\title{
Prevalence and risk factors of myocardial and acute kidney injury following radical nephrectomy with vena cava thrombectomy: a retrospective cohort study
}

Yi-Bin Hua ${ }^{1}$, Xue Li ${ }^{1}$ and Dong-Xin Wang ${ }^{1,2^{*}}$

\begin{abstract}
Background: Radical nephrectomy with thrombectomy is the mainstay treatment for patients with renal cell carcinoma with vena cava thrombus. But the procedure is full of challenge, with high incidence of major complications and mortality. Herein, we investigated the incidence and predictors of myocardial injury and acute kidney injury (AKI) in patients following radical nephrectomy with inferior vena cava thrombectomy.
\end{abstract}

Methods: Patients who underwent nephrectomy with thrombectomy between January 2012 and June 2020 were retrospectively reviewed. Myocardial injury was diagnosed when peak cardiac troponin I was higher than $0.03 \mathrm{ng} /$ ml. AKI was diagnosed according to the Kidney Disease: Improving Global Outcomes (KDIGO) criteria. Multivariable logistic regression models were used to identify predictors of myocardial injury or AKI after surgery.

Results: A total of 143 patients were included in the final analysis. Myocardial injury and AKI occurred in 37.8 and $42.7 \%$ of patients after this surgery, respectively. Male sex (odds ratio [OR] 0.27, 95\% confidence interval [CI] 0.10-0.71; $P=0.008$ ) was associated with a lower risk, whereas high level Mayo classification (compared with Mayo level I $+\|$, Mayo level III + IV: OR 4.21, 95\% Cl 1.42-12.4; $P=0.009$ ), acute normovolemic hemodilution before surgery (OR 2.66, $95 \% \mathrm{Cl} 1.10-6.41 ; P=0.029$ ), long duration of intraoperative tachycardia (per $20 \mathrm{~min}$ : OR 1.49, 95\% Cl 1.10-2.16; $P=0.036$ ), and long duration of surgery (per $1 \mathrm{~h}, \mathrm{OR} 1.48,95 \% \mathrm{Cl} 1.03-2.16, P=0.009$ ) were associated with a higher risk of myocardial injury. High body mass index $(\mathrm{OR} 1.18,95 \% \mathrm{Cl} 1.06-1.33 ; P=0.004)$ and long duration of intraoperative hypotension (per $20 \mathrm{~min}$ : OR 1.30,95\% Cl 1.04-1.64; $P=0.024$ ) were associated with a higher risk, whereas selective renal artery embolism before surgery $(\mathrm{OR} 0.20,95 \% \mathrm{Cl} 0.07-0.59, P=0.004)$ was associated with a lower risk of AKI.

Conclusion: Myocardial injury and AKI were common in patients recovering from radical nephrectomy with inferior vena cava thrombectomy. Whether interventions targeting the above modifiable factors can improve outcomes require further studies.

Keywords: Renal cell carcinoma, Radical nephrectomy, Inferior vena cava thrombectomy, Myocardial injury, Acute kidney injury

*Correspondence: dxwang65@bjmu.edu.cn; wangdongxin@hotmail.com ${ }^{1}$ Department of Anesthesiology and Critical Care Medicine, Peking University First Hospital, Beijing 100034, China

Full list of author information is available at the end of the article

\section{Background}

Renal cell carcinoma (RCC) has a propensity to develop local extension into the venous system. About 4 to $10 \%$ of newly diagnosed RCC cases have venous tumor thrombus [1-4]. In a study of 540 patients with RCC and original author(s) and the source, provide a link to the Creative Commons licence, and indicate if changes were made. The images or other third party material in this article are included in the article's Creative Commons licence, unless indicated otherwise in a credit line to the material. If material is not included in the article's Creative Commons licence and your intended use is not permitted by statutory regulation or exceeds the permitted use, you will need to obtain permission directly from the copyright holder. To view a copy of this licence, visit http://creativecommons.org/licenses/by/4.0/. The Creative Commons Public Domain Dedication waiver (http://creativeco mmons.org/publicdomain/zero/1.0/) applies to the data made available in this article, unless otherwise stated in a credit line to the data. 
venous tumor thrombus, $64.6 \%$ had renal venous thrombus (Mayo level 0) and 35.4\% had inferior vena cava (IVC) thrombus (12.2\% Mayo level I, 14.3\% Mayo level II, 5.2\% Mayo level III, and 3.7\% Mayo level IV, respectively) [5]. For patients with RCC and IVC tumor thrombus, radical nephrectomy with thrombectomy remains the mainstay treatment and offers reasonable long-term survival [6-8]. However, perioperative management of such patients is a great challenge for both surgeons and anesthesiologists [9-12]. Previous studies reported that major complications occurred in 6 to $34 \%$ and mortality occurred in 0 to $10.5 \%$ of patients following radical nephrectomy with IVC thrombectomy [13-15].

As a challenging surgery, nephrectomy with IVC thrombectomy may also put patients at risk of myocardial and acute kidney injury (AKI). Myocardial injury is defined as troponin elevation [16] and occurred in 8 to $16 \%$ patients after non-cardiac surgery $[17,18]$. Although usually asymptomatic and without electrocardiographic and imagining changes [19], the occurrence of myocardial injury is associated with worse outcomes including increased 30-day and 1-year mortality [18, 20, 21]. AKI, characterized by oliguria and increased serum creatinine and other biomarkers [22, 23], is also common after major non-cardiac surgery with an incidence from 6.8 to $39.3 \%[24,25]$ and up to $61.6 \%$ after radical nephrectomy [26]. Development of postoperative AKI is associated with prolonged hospital stay, long-term decline of renal function, and high mortality [27-29]. However, few studies have focused on the incidence and risk factors of myocardial injury and AKI after radical nephrectomy with IVC thrombectomy. In a small sample size study of 76 patients, $53.9 \%$ developed AKI after surgery; long IVC clamping time was identified as a potentially modifiable risk factor [30].

A better understanding of the occurrence and underlying risk factors of myocardial injury and AKI may help us to prevent these harmful complications after surgery for RCC and IVC thrombus. This retrospective study aimed to investigate the incidence and risk factors of myocardial injury and AKI in patients undergoing radical nephrectomy with IVC thrombectomy.

\section{Methods and materials}

\section{Ethics and consent}

This retrospective cohort study was performed in Peking University First Hospital. The study protocol was approved by the Biomedical Research Ethics Committee of Peking University First Hospital (2019-205). Considering that all data were collected from the hospital electronic medical record system and no patient follow-ups were performed, the Ethics Committee agreed to waive written informed consents. All personal data were kept strictly confidential. The study was performed in accordance with Strengthening the Reporting of Observational Studies in Epidemiology (STROBE) criteria (see Additional file 1: STROBE checklist).

\section{Study population}

Patients who underwent radical nephrectomy with tumor thrombectomy from January 2012 to June 2020 were screened for study inclusion. The inclusion criteria were adult (age $\geq 18$ years) patients who were diagnosed with RCC and IVC tumor thrombus (i.e., Mayo levels I to IV) and underwent radical nephrectomy with IVC thrombectomy. Patients were excluded if they had incomplete data for primary outcome assessment (i.e., no serum troponin or creatinine test results after surgery) in the electronic medical records, were classified as Mayo level 0 (tumor thrombus limited to the renal vein), underwent concomitant cardiac surgery, or turned out to be non-renal carcinomas according to postsurgical pathological report.

\section{Perioperative management}

All patients received contrast-enhanced abdominal computed tomography or magnetic resonance imaging within about 2 weeks before surgery. The thrombus level was classified according to the Mayo classification: Level 0 indicates tumor thrombus limited within the renal vein; Level I, tumor thrombus extending into the IVC to no more than $2 \mathrm{~cm}$ above the renal vein; level II, thrombus extending into the IVC to more than $2 \mathrm{~cm}$ above renal vein but below the hepatic veins; level III, thrombus extending into the IVC to above the hepatic vein but not to the diaphragm; and level IV, thrombus extending into the supradiaphragmatic IVC or right atrium [5]. Selective renal arterial embolization was performed preoperatively depending on patients' condition.

In the operating room, routine monitoring included electrocardiogram (ECG), non-invasive blood pressure, pulse oxygen saturation, end-tidal concentration of carbon dioxide and inhaled anesthetics, nasopharyngeal temperature, Bispectral Index, and urine output. Invasive blood pressure was monitored through an intraarterial cannula with or without dynamic parameter (such as stroke volume variation or pulse pressure variation) monitoring. A central venous line was established through which acute normovolemic hemodilution was performed after anesthesia induction when considered necessary. For patients with tumor thrombi of Mayo level III or above, transesophageal echocardiogram was used to monitor the position of the tumor thrombus.

General anesthesia was performed for all patients. Anesthesia was induced with intravenous propofol/etomidate, opioids (sufentanil/remifentanil) and muscle relaxants (rocuronium or cisatracurium), and 
maintained with intravenous infusion of propofol and sufentanil/remifentanil, with or without inhalational nitrous oxide and/or sevoflurane. Muscle relaxation was maintained with rocuronium or cisatracurium. Regional block was performed and dexmedetomidine was administered at the discretion of anesthesiologists. Acute normovolemic hemodilution was performed after anesthesia induction but before surgical incision. The volume of collected blood was calculated so that the hematocrit was maintained above $27 \%$ after hemodilution [31]. Balanced crystalloid fluid was routinely infused. Normal saline was only used as a carrier for antibiotics and other drugs. For patients with large blood loss, artificial colloid was infused for volume resuscitation; blood products were transfused in order to maintain hemoglobin $>7 \mathrm{~g} / \mathrm{dl}$ and normal coagulation. The target of hemodynamic management was to maintain blood pressure and heart rate within $30 \%$ from baseline and a urine output $>0.5 \mathrm{ml} / \mathrm{kg} / \mathrm{h}$.

Surgery was performed via laparoscopic, open or combined approaches, depending on the condition of tumor and the decision of surgeons. For patients with Mayo level I thrombi, surgeries were usually performed under partial IVC clamping. Patients with Mayo level II thrombi usually required complete clamping of IVC and renal vein. For patients with tumor thrombi of Mayo level III or above, additional cross-clamping of hepatic hilar might be necessary or cardiopulmonary bypass $(\mathrm{CPB})$ was performed with systemic heparinization. Due to the nature of cancer surgery, intraoperative blood salvage was not performed unless for patients with massive bleeding, during which case the salvaged blood would be transfused after obtaining written informed consents.

After surgery, patients with intraoperative hemodynamic instability, massive bleeding, or CPB were admitted to the intensive care unit (ICU); otherwise, they were admitted to the post-anesthesia care unit for at least $30 \mathrm{~min}$ and then transferred back to the general wards. Crystalloid solutions (containing electrolytes, glucose, and other non-electrolyte solutes) were infused. Blood products (packed red blood cells, fresh frozen plasma, and/or albumin) were administered as necessary. As a routine practice, serum levels of cardiac troponin I (cTnI) and creatinine were monitored at least once during the first three postoperative days or longer when considered necessary. Patient-controlled analgesia (PCA) was provided for postoperative analgesia. Nonsteroidal anti-inflammatory drugs were allowed for those without contraindications. Other postoperative care was provided per routine.

\section{Data acquisition and outcomes}

Data were collected from the electronic medical record system of the hospital. Baseline data included demographic characteristics, comorbidities, smoking and surgical history, main laboratory test results (including baseline cTnI and creatinine), and location and maximal diameter of the tumor. Charlson Comorbidity Index was calculated. American Society of Anesthesiologists classification and New York Heart Association classification were evaluated. Mayo classification was obtained from surgical records.

Intraoperative data included the conduct of selective renal arterial embolization, type and duration of anesthesia, medication during anesthesia, performance of $\mathrm{ANH}$, intraoperative levels of hemoglobin and lactic acid, occurrence of hemodynamic fluctuation, fluid infusion, blood loss and allogeneic blood transfusion, urine output, type and duration of surgery, combined surgery, use of IVC clamping and hepatic hilar clamping, use of $\mathrm{CPB}$, as well as administration of PCA after surgery. Hemodynamic data were obtained from the anesthesia information system, which captured parameters automatically every $10 \mathrm{~s}$ throughout the intraoperative period.

The primary outcomes were myocardial injury and AKI after surgery. The results of serum cTnI and creatinine during early postoperative days were collected. Myocardial injury was diagnosed when peak cTnI was higher than the 99th percentile upper reference limit $(>0.03 \mathrm{ng} / \mathrm{ml})$ or, for patients with preoperative cTnI above normal, an absolute increase of $\geq 0.03 \mathrm{ng} / \mathrm{ml}$ [16-18]. Acute myocardial infarction (AMI) was diagnosed when myocardial injury was accompanied by clinical evidence of acute myocardial ischemia (symptoms, ECG changes, or imaging findings) [16]. AKI was diagnosed according to the Kidney Disease: Improving Global Outcomes (KDIGO) criteria [32], i.e., an increase in serum creatinine $\geq 26.5 \mu \mathrm{mol} / 1 \quad(\geq 0.3 \mathrm{mg} /$ dl) within $48 \mathrm{~h}$, or an increase in serum creatinine to $\geq 1.5$ times baseline within 7 days after surgery. For patients who developed AKI, the severity was classified into 3 stages. Stage 1 indicates 1.5 to 1.9 times baseline or $\geq 26.5 \mu \mathrm{mol} / 1$ increase; stage 2 indicates 2.0 to 2.9 times baseline; stage 3 indicates 3.0 times baseline or an increase in serum creatinine to $\geq 353.6 \mu \mathrm{mol} / \mathrm{l}$ or requirement of renal replacement therapy [32].

Other postoperative outcomes, including ICU admission after surgery, the development of other postoperative complications, length of hospital stay after surgery, and in-hospital mortality were also collected. For patients admitted to the ICU, the duration of mechanical ventilation and length of ICU stay were also recorded. 


\section{Statistical analyses}

Continuous variables are presented as the mean \pm standard deviation or median (interquartile range [IQR]). Data were compared using the student's t test (normal distribution) or Mann-Whitney U test (non-normal distribution). Categorical variables are presented as number of patients (percentage). Data were analyzed using the Chi-squared test, Chi-square test with continuity correction or Fisher's exact test. Time-to-event variables are presented as median (95\% CI). Data were analyzed using Kaplan-Meier survival analysis, with difference between group compared with log-rank test. Missing data were not replaced.

Univariate logistic regression analyses were performed to screen factors in association with the occurrence of myocardial injury and AKI. Linear regression was used to test the multicollinearity among variables, a variance inflation factor of $>10$ was considered the existence of multicollinearity. Independent variables with $P<0.20$ in univariable analyses or were considered clinically important were included in multivariable logistic regression models to identify factors that were significantly associated with the occurrence of myocardial injury or AKI after surgery. HosmerLemeshow test was used to assess the goodness-of-fit of the models. A two-sided $P$ value of $<0.05$ was considered statistically significant. All data were analyzed using SPSS (version 25.0; IBM SPSS, Inc., Chicago, IL, USA).

We did not estimate sample size in advance. However, according to the "ten events per variable" rule and the number of independent variables included in the final multivariable logistic regression models, the number of patients with outcome events was sufficient to guarantee the stability of the regression estimates.

\section{Results}

\section{Patients}

A total of 199 patients underwent radical nephrectomy with tumor thrombectomy from January 1, 2012 to June 30, 2020. Among these, 36 patients were excluded due to Mayo level 0 thrombi and missing data, 10 patients were excluded due to the Mayo level 0 thrombi, 6 patients were excluded for concomitant cardiac surgeries, and 4 patients were excluded for non-renal carcinomas. The remaining 143 patients were included in the final analysis (Fig. 1).

When compared with patients without myocardial injury, those who developed myocardial injury had lower male sex ratio, higher baseline cTnI level, and higher percentage with Mayo level IV thrombi (Table 1). During anesthesia, those who developed myocardial injury received less nitrous oxide inhalation but more $\mathrm{ANH}$, had lower hemoglobin but higher lactic acid level, developed more and longer hypotension and tachycardia; they underwent longer anesthesia and surgery, received more fluid infusion and blood products, had more urine output and blood loss, endured more IVC/hepatic hilum clamping and $\mathrm{CPB}$, and were given more PCA (Table 2).

When compared with patients without AKI, those who developed AKI had higher body mass index (BMI) and lower baseline creatinine level (Table 1). During anesthesia, they had higher lactic acid level and underwent more combined non-renal surgery (mainly splenectomy and cholecystectomy) (Table 2).

\section{Postoperative outcomes}

Of the included patients, 54 (37.8\%) developed myocardial injury and 11 (7.7\%) developed AMI. The majority of myocardial injury occurred in patients with Mayo levels III and IV thrombi and during the first day after surgery (Fig. 2a and b). When compared with patients

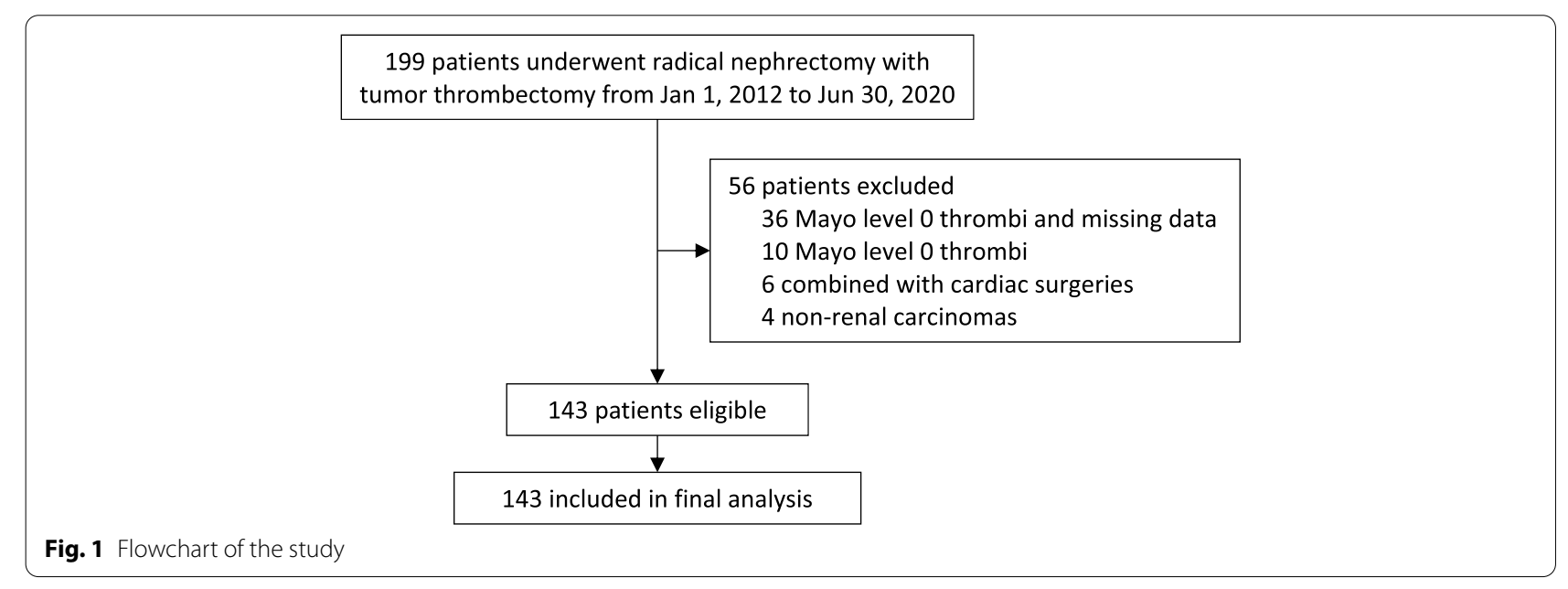


Table 1 Baseline data

\begin{tabular}{|c|c|c|c|c|c|c|}
\hline & $\begin{array}{l}\text { Non-myocardial injury } \\
(n=89)\end{array}$ & $\begin{array}{l}\text { Myocardial injury } \\
(n=54)\end{array}$ & $P$ value & Non-AKI $(n=82)$ & AKI $(n=61)$ & $P$ value \\
\hline Age, year & $59(50,67)$ & $55(48,64)$ & 0.092 & $56(44,68)$ & $58(46,70)$ & 0.433 \\
\hline Body mass index, $\mathrm{kg} / \mathrm{m}^{2}$ & $24.2(22.6,27.1)$ & $23.9(21.6,26.8)$ & 0.441 & $24.0(20.7,27.3)$ & $25.9(19.6,32.2)$ & 0.021 \\
\hline Male sex & $71(79.8 \%)$ & $34(63.0 \%)$ & 0.027 & $63(76.8 \%)$ & $42(68.9 \%)$ & 0.286 \\
\hline \multicolumn{7}{|l|}{ Comorbidities } \\
\hline Stroke & $3(3.4 \%)$ & $4(7.4 \%)$ & 0.278 & $2(2.4 \%)$ & $5(8.2 \%)$ & 0.115 \\
\hline Hypertension & $36(40.4 \%)$ & $22(40.7 \%)$ & 0.973 & $31(37.8 \%)$ & $27(44.3 \%)$ & 0.437 \\
\hline Chronic heart disease ${ }^{a}$ & $6(6.7 \%)$ & $6(11.1 \%)$ & 0.361 & $6(7.3 \%)$ & $6(9.8 \%)$ & 0.591 \\
\hline Diabetes Mellitus & $16(18.0 \%)$ & $9(16.7 \%)$ & 0.841 & $17(20.7 \%)$ & $8(13.1 \%)$ & 0.236 \\
\hline COPD & $4(4.5 \%)$ & $0(0.0 \%)$ & 0.290 & $4(4.9 \%)$ & $0(0.0 \%)$ & 0.216 \\
\hline Elevated transaminase ${ }^{\mathrm{b}}$ & $6(6.7 \%)$ & $5(9.3 \%)$ & 0.584 & $7(8.5 \%)$ & $4(6.6 \%)$ & 0.660 \\
\hline $\begin{array}{l}\text { Abnormal kidney func- } \\
\text { tion }{ }^{c}\end{array}$ & $9(10.1 \%)$ & $2(3.7 \%)$ & 0.163 & $8(9.8 \%)$ & $3(4.9 \%)$ & 0.283 \\
\hline Smoking history & $29(32.6 \%)$ & $18(33.3 \%)$ & 0.926 & $25(30.5 \%)$ & $22(36.1)$ & 0.482 \\
\hline Previous surgery & $15(16.9 \%)$ & $10(18.5 \%)$ & 0.799 & $18(22.0 \%)$ & $7(11.5 \%)$ & 0.103 \\
\hline $\begin{array}{l}\text { Charlson Comorbidity } \\
\text { Index }{ }^{d}\end{array}$ & $2(2,2)$ & $2(2,2)$ & 0.748 & $2(2,2)$ & $2(2,2)$ & 0.931 \\
\hline \multicolumn{7}{|l|}{ Laboratory tests } \\
\hline Hemoglobin, g/L & $119(105,135)$ & $110(98,130)$ & 0.429 & $112(96,134)$ & $117(108,131)$ & 0.327 \\
\hline Albumin, g/L & $37.8(35.1,41.2)$ & $36.5(33.8,40.7)$ & 0.227 & $37.6(35.1,41.4)$ & $37.6(34.4,40.8)$ & 0.728 \\
\hline $\mathrm{cTnl}, \mathrm{ng} / \mathrm{ml}$ & $0.004(0.001,0.010)[63]$ & $0.010(0.007,0.010)[27]$ & 0.026 & $0.010(0.003,0.010)[56]$ & $0.007(0.001,0.010)[34]$ & 0.296 \\
\hline$c T n l>0.03 \mathrm{ng} / \mathrm{ml}{ }^{\mathrm{e}}$ & $2(2.2 \%)$ & $2(3.7 \%)$ & $>0.999$ & $2(2.4 \%)$ & $2(3.3 \%)$ & 0.969 \\
\hline Creatinine, $\mu \mathrm{mol} / \mathrm{L}$ & $96.0(80.3,114.0)$ & $90.5(80.1,105.3)$ & 0.422 & $101.0(83.0,117.0)$ & $88.3(74.2,104.0)$ & 0.004 \\
\hline ASA classification & & & 0.095 & & & 0.732 \\
\hline I & $8(9.0 \%)$ & $1(1.9 \%)$ & & $5(6.1 \%)$ & $4(6.6 \%)$ & \\
\hline$\|$ & $59(66.3 \%)$ & $32(59.3 \%)$ & & $54(65.9 \%)$ & $37(60.7 \%)$ & \\
\hline III & $22(24.7 \%)$ & $20(37.0 \%)$ & & $22(26.8 \%)$ & $20(32.8 \%)$ & \\
\hline IV & $0(0 \%)$ & $1(1.9 \%)$ & & $1(1.2 \%)$ & $0(0 \%)$ & \\
\hline NYHA classification & & & 0.407 & & & 0.531 \\
\hline 1 & $77(86.5 \%)$ & $47(87.0 \%)$ & & 70 (85.4\%) & $54(88.5 \%)$ & \\
\hline$\|$ & $12(13.5 \%)$ & $6(11.1 \%)$ & & $12(14.6 \%)$ & $6(9.8 \%)$ & \\
\hline ॥ा & $0(0 \%)$ & $1(1.9 \%)$ & & $0(0 \%)$ & $1(1.6 \%)$ & \\
\hline Right renal tumor (vs. left) & $61(68.5 \%)$ & $42(77.8 \%)$ & 0.233 & $60(73.2 \%)$ & $43(70.5 \%)$ & 0.724 \\
\hline $\begin{array}{l}\text { Maximal tumor diameter, } \\
\mathrm{cm}\end{array}$ & & & 0.261 & & & 0.088 \\
\hline 0 to 5 & $8(9.0 \%)$ & $3(5.6 \%)$ & & $6(7.3 \%)$ & $5(8.2 \%)$ & \\
\hline$>5$ to 10 & $42(47.2 \%)$ & $33(61.1 \%)$ & & $37(45.1 \%)$ & $38(62.3 \%)$ & \\
\hline$>10$ & $39(43.8 \%)$ & $18(33.3 \%)$ & & $39(47.6 \%)$ & $18(29.5 \%)$ & \\
\hline Mayo classification ${ }^{f}$ & & & $<0.001$ & & & 0.531 \\
\hline 1 & $18(20.2 \%)$ & $4(7.4 \%)$ & & $14(17.1 \%)$ & $8(13.1 \%)$ & \\
\hline$\|$ & $27(30.3 \%)$ & $2(3.7 \%)$ & & $19(23.2 \%)$ & $10(16.4 \%)$ & \\
\hline III & $40(44.9 \%)$ & $23(42.6 \%)$ & & 35 (42.7\%) & $28(45.9 \%)$ & \\
\hline IV & $4(4.5 \%)$ & $25(46.3 \%)$ & & $14(17.1 \%)$ & $15(24.6 \%)$ & \\
\hline $\begin{array}{l}\text { Interval from contrast- } \\
\text { enhanced examination, } \\
\text { day }{ }^{g}\end{array}$ & $13(7,20)$ & $13(5,20)$ & 0.997 & $12(4,18)$ & $14(7,24)$ & 0.181 \\
\hline
\end{tabular}

Data are median (interquartile range) or number (\%). Numbers in square brackets indicate patients with missing data. $P$ values in bold indicate $<0.05$ COPD chronic obstructive pulmonary injury, cTn/ cardiac troponin I, ASA American Society of Anesthesiologists, NYHA New York Heart Association

a Including coronary artery disease, cardiac valve disease, or any type of arrhythmia requiring therapy

b Serum aspartate aminotransferase and/or alanine aminotransferase were higher than the upper normal limit

c Indicating serum creatinine $\geq 133 \mu \mathrm{mol} / \mathrm{L}$

d According to the 1987 version without age correction 
Table 1 (continued)

\footnotetext{
e Patients without preoperative cardiac troponin I results were regarded as within normal range

${ }^{f}$ Level I, tumor thrombus extending into the IVC to no more than $2 \mathrm{~cm}$ above the renal vein; level II, thrombus extending into the IVC to more than $2 \mathrm{~cm}$ above renal vein but below the hepatic veins; level III, thrombus extending into the IVC to above the hepatic vein but not to the diaphragm; and level IV, thrombus extending into the supradiaphragmatic IVC or right atrium

${ }^{g}$ Time interval from contrast-enhanced examination to surgery
}

without myocardial injury, those with myocardial injury developed more AKI (57.4\% [31/54] vs. 33.7\% [30/89], $P=0.005)$ and other complications (24.1\% [13/54] vs. $1.1 \%[1 / 89], P=0.001)$, had more ICU admission (94.4\% [51/54] vs. $64.0 \%$ [57/89], $P<0.001)$, and stayed longer in hospital after surgery (hazard ratio [HR] 1.94, 95\% CI 1.37-2.74, $P<0.001)$. Among patients admitted to the ICU, those with myocardial injury had more endotracheal intubation (90.7\% [49/54] vs. $49.4 \%$ [44/89], $P<0.001$ ), endured longer mechanical ventilation (HR 2.40, 95\% CI 1.67-3.44, $P<0.001)$, and stayed longer in the ICU (HR 2.39, 95\% CI 1.68-3.41, $P<0.001)$ than those without (Table 3).

Of the included patients, 61 (42.7\%) developed AKI; of these, $53(86.9 \%)$ had stage 1, $6(9.8 \%)$ had stage 2 , and 2 (3.3\%) had stage 3 AKI. The occurrence of AKI was not related with Mayo level of thrombi, but the majority occurred within the first two postoperative days (Fig. 2c and $\mathrm{d}$ ). When compared with patients without AKI, patients with AKI developed more myocardial injury (50.8\% [31/61] vs. $28.0 \%$ [23/82], $P=0.005)$, more AMI (13.1\% [8/61] vs. $3.7 \%$ [3/82], $P=0.036)$, and more other complications $(19.7 \%[12 / 61]$ vs. $2.4 \%[2 / 82], P=0.005)$, and stayed longer in hospital after surgery (HR 1.47, 95\% CI 1.04-2.06, $P=0.027$ ). Although ICU admission rate did not differ between patients with or without AKI, those with AKI stayed longer in the ICU after surgery (HR 1.41, 95\% CI 1.01-1.98, $P=0.046$; Table 3).

\section{Predictors of myocardial injury}

Univariable analyses identified 25 factors with $P$ values $<0.20$ (see Additional file 2: Supplement Table). After testing of multicollinearity, 12 factors were included in the multivariable logistic regression model. Final analysis identified five independent predictors of myocardial injury after surgery. Of these, male sex (OR 0.27, 95\% CI $0.10-0.71 ; P=0.008)$ was associated with a lower risk, whereas high Mayo level (compared with Mayo level I+ II, Mayo level III+IV: OR 4.21, 95\% CI 1.42-12.4; $P=0.009$ ), acute normovolemic hemodilution before surgery (OR 2.66, 95\% CI 1.10-6.41; $P=0.029$ ), long duration of intraoperative tachycardia (per $20 \mathrm{~min}$ : OR 1.49, 95\% CI 1.03-2.16; $P=0.036$ ), and long duration of surgery (per $1 \mathrm{~h}$, OR $1.48,95 \% \mathrm{CI} 1.03-2.16 ; P=0.009$ ) were associated with a higher risk of myocardial injury (Table 4).

\section{Predictors of AKI}

Univariable analyses identified 13 factors with $P$ values $<0.20$ (see Additional file 2: Supplement Table). After testing of multicollinearity, 12 factors together with age and Mayo classification were included in the multivariable logistic regression model. Final analysis identified three independent predictors of AKI after surgery. Of these, high BMI (OR 1.18, 95\% CI 1.06-1.33; $P=0.004$ ) and long duration of intraoperative hypotension (per 20 min: OR 1.30, 95\% CI 1.04-1.64; $P=0.024$ ) were associated with a higher risk, whereas selective renal artery embolism before surgery (OR 0.20, 95\% CI $0.07-$ $0.59 ; P=0.005)$ was associated with a lower risk of AKI (Table 5).

\section{Discussion}

Results of this retrospective study showed that myocardial injury occurred in $37.8 \%$ and AKI occurred in $42.7 \%$ of patients following radical nephrectomy with IVC thrombectomy. Patients with myocardial injury or AKI had worse perioperative outcomes, including more other complications and longer length of hospital stay. Male sex was associated with a lower risk, whereas high Mayo level, acute normovolemic hemodilution, long intraoperative tachycardia, and long surgery were associated with a higher of myocardial injury. High BMI and long intraoperative hypotension were associated with a higher risk, whereas preoperative renal artery embolism was associated with a lower risk of AKI.

As far as we know, this is the first study exploring the incidence and risk factors of myocardial injury after radical nephrectomy with IVC thrombectomy. As expected, the incidence of myocardial injury in our patients was higher when compared with the majority of previous results. For example, the VISION study reported an incidence of $8 \%$ [17] and Puelacher et al. [18] reported an incidence of $16 \%$ of myocardial injury after noncardiac surgery. However, myocardial injury occurred in up to $40.4 \%$ of patients after liver transplantation [33], which is close to our results. The high incidence of myocardial injury in our patients can be attributed to the significant hemodynamic fluctuation during surgery. Indeed, among our patients, $83.2 \%$ underwent complete IVC clamping, $27.3 \%$ underwent hepatic hilum clamping, and $26.6 \%$ required $\mathrm{CPB}$. It is not surprising that $89.5 \%$ of our patients endured intraoperative hypotension (mean 
Table 2 Intra- and postoperative data

\begin{tabular}{|c|c|c|c|c|c|c|}
\hline & $\begin{array}{l}\text { Non-myocardial } \\
\text { injury }(n=89)\end{array}$ & Myocardial injury $(n=54)$ & $P$ value & Non-AKI $(n=82)$ & AKI $(n=61)$ & $P$ value \\
\hline Selective renal arterial embolization & $19(21.3 \%)$ & $8(14.8 \%)$ & 0.333 & $20(24.4 \%)$ & $7(11.5 \%)$ & 0.051 \\
\hline Type of anesthesia & & & 0.784 & & & 0.229 \\
\hline General & $76(85.4 \%)$ & $47(87.0 \%)$ & & $73(89.0 \%)$ & $50(82.0 \%)$ & \\
\hline Combined regional-general ${ }^{\mathrm{a}}$ & $13(14.6 \%)$ & $7(13.0 \%)$ & & $9(11.0 \%)$ & $11(18.0 \%)$ & \\
\hline \multicolumn{7}{|l|}{ Medication during anesthesia } \\
\hline Nitrous oxide & $69(77.5 \%)$ & $33(61.1 \%)$ & 0.035 & $61(74.4 \%)$ & $41(67.2 \%)$ & 0.348 \\
\hline Sevoflurane & $51(57.3 \%)$ & $28(51.9 \%)$ & 0.525 & $41(50.0 \%)$ & $38(62.3 \%)$ & 0.144 \\
\hline Dexmedetomidine & $41(46.1 \%)$ & $27(50.0 \%)$ & 0.648 & $43(52.4 \%)$ & $25(41.0 \%)$ & 0.175 \\
\hline ANH before surgery ${ }^{b}$ & $25(28.1 \%)$ & $25(46.3 \%)$ & 0.027 & $25(30.5 \%)$ & $25(41.0 \%)$ & 0.193 \\
\hline Hemoglobin after ANH, g/L ${ }^{\mathrm{C}}$ & $98(86,110)$ & $102(94,109)$ & 0.403 & $105(88,111)$ & $98(91,109)$ & 0.466 \\
\hline Lactic acid after ANH, mmol/ $\mathrm{L}^{\mathrm{c}}$ & $1.0(0.6,1.4)$ & $0.8(0.5,1.2)[5]$ & 0.292 & $0.9(0.5,1.2)[2]$ & $0.9(0.7,1.3)[3]$ & 0.466 \\
\hline \multicolumn{7}{|l|}{ Extremes of arterial blood gas analyses } \\
\hline Lowest hemoglobin, g/L & $95(77,112)$ & $74(61,88)$ & $<0.001$ & $87(62,112)$ & $90(64,116)$ & 0.632 \\
\hline Highest lactic acid, mmol/L & $1.2(0.9,1.6)$ & $1.7(1.0,2.5)$ & 0.004 & $1.5(0.6,2.2)$ & $2.0(0.1,3.9)$ & 0.044 \\
\hline Highest lactic acid $>2 \mathrm{mmol} / \mathrm{L}$ & $15(16.9 \%)$ & $21(38.9 \%)$ & 0.002 & $17(20.7 \%)$ & $19(31.1 \%)$ & 0.156 \\
\hline \multicolumn{7}{|l|}{ Hemodynamic change during anesthesia } \\
\hline Hypotension ${ }^{d}$ & $76(85.4 \%)$ & $52(96.3 \%)$ & 0.039 & $73(89.0 \%)$ & $55(90.2 \%)$ & 0.826 \\
\hline Duration of hypotension, min & $8(1,23)$ & $37(9,60)$ & $<0.001$ & $12(1,35)$ & $18(3,54)$ & 0.141 \\
\hline Tachycardia $^{e}$ & $45(50.6 \%)$ & $44(81.5 \%)$ & $<0.001$ & $46(56.1 \%)$ & $43(70.5 \%)$ & 0.079 \\
\hline Other tachyarrhythmia ${ }^{f}$ & $2(2.2 \%)$ & $8(14.8 \%)$ & 0.004 & $6(7.3 \%)$ & $4(6.6 \%)$ & 0.860 \\
\hline Duration of tachycardia, min ${ }^{e, f}$ & $0.2(0.0,3.3)$ & $6.2(0.3,22.2)$ & $<0.001$ & $0.9(0.0,8.1)$ & $2.2(0.0,9.7)$ & 0.303 \\
\hline Bradycardia $^{9}$ & $11(12.4 \%)$ & $16(29.6 \%)$ & 0.011 & $14(17.1 \%)$ & $13(21.3 \%)$ & 0.522 \\
\hline Total fluid infused, ml & $4000(2800,5600)$ & $5550(4050,7300)$ & 0.001 & $4800(3300,6125)$ & $4900(2800,6700)$ & 0.637 \\
\hline Balanced crystalloid, ml & $2400(2000,3500)$ & $2650(1925,3700)$ & 0.289 & $2575(2000,3525)$ & $2500(1700,3700)$ & 0.219 \\
\hline Normal saline, ml & $100(100,100)$ & $100(100,200)$ & 0.417 & $100(100,125)$ & $100(100,100)$ & 0.542 \\
\hline Hydroxyethyl starch, ml & $500(0,500)$ & $500(0,1000)$ & 0.428 & $500(0,625)$ & $500(0,1000)$ & 0.528 \\
\hline Succinylated gelatin, ml & $500(0,500)$ & $500(0,500)$ & 0.503 & $500(0,500)$ & $500(0,500)$ & 0.729 \\
\hline Urine output, $\mathrm{ml}$ & $550(300,900)$ & $850(500,1750)$ & $<0.001$ & $700(338,1263)$ & $600(300,1100)$ & 0.336 \\
\hline Estimated blood loss, ml & $500(200,1000)$ & $1000(500,2100)$ & $<0.001$ & $600(300,1525)$ & $800(200,1450)$ & 0.453 \\
\hline Allogeneic blood transfusion & $38(42.7 \%)$ & $43(79.6 \%)$ & $<0.001$ & $44(53.7 \%)$ & $37(60.7 \%)$ & 0.404 \\
\hline Volume of red blood cells, ml & $0(0,400)$ & $400(0,800)$ & 0.306 & $100(0,800)$ & $400(0,800)$ & 0.490 \\
\hline Fresh frozen plasma & $28(31.5 \%)$ & $34(63.0 \%)$ & $<0.001$ & $34(41.5 \%)$ & $28(45.9 \%)$ & 0.596 \\
\hline Platelets concentrate & $2(2.2 \%)$ & $21(38.9 \%)$ & $<0.001$ & $9(11.0 \%)$ & $14(23.0 \%)$ & 0.054 \\
\hline Intraoperative fluid balance, ml & $2730(2000,3930)$ & $3350(2060,4100)$ & 0.202 & $3100(2080,3960)$ & $2830(1900,4160)$ & 0.636 \\
\hline Duration of anesthesia, $\mathrm{h}$ & $5.7(4.4,7.1)$ & $7.9(6.5,9.5)$ & $<0.001$ & $6.6(4.8,7.9)$ & $6.4(4.9,8.6)$ & 0.909 \\
\hline Type of surgery & & & 0.078 & & & 0.081 \\
\hline Laparoscopic & $16(18.0 \%)$ & $3(5.6 \%)$ & & $7(8.5 \%)$ & $12(19.7 \%)$ & \\
\hline Open & $38(42.7 \%)$ & $23(42.6 \%)$ & & $40(48.8 \%)$ & $21(34.4 \%)$ & \\
\hline Laparoscopic combined open & 35 (39.3\%) & $28(51.9 \%)$ & & $35(42.7 \%)$ & $28(45.9 \%)$ & \\
\hline Combined with non-renal surgery ${ }^{h}$ & $2(2.2 \%)$ & $1(1.9 \%)$ & 0.873 & $0(0 \%)$ & $3(4.9 \%)$ & 0.042 \\
\hline Complete IVC clamping & $69(77.5 \%)$ & $50(92.6 \%)$ & 0.019 & $68(82.9 \%)$ & $51(83.6 \%)$ & 0.914 \\
\hline Hepatic hilum clamping & $10(11.2 \%)$ & $29(53.7 \%)$ & $<0.001$ & $24(29.3 \%)$ & $15(24.6 \%)$ & 0.534 \\
\hline Use of cardiopulmonary bypass & $6(6.7 \%)$ & $32(59.3 \%)$ & $<0.001$ & $20(24.4 \%)$ & $18(29.5 \%)$ & 0.493 \\
\hline Duration of cardiopulmonary bypass, $\min ^{i}$ & $0(0,0)$ & $21(0,31)$ & $<0.001$ & $0(0,3.5)$ & $0(0,20)$ & 0.478 \\
\hline Duration of surgery, $\mathrm{h}$ & $4.2(3.1,5.5)$ & $6.3(5.0,7.7)$ & $<0.001$ & $5.0(3.5,5.9)$ & $5.0(3.5,7.0)$ & 0.746 \\
\hline Use of PCA after surgery & $81(91.0 \%)$ & $54(100.0 \%)$ & 0.023 & $75(91.5 \%)$ & $60(98.4 \%)$ & 0.138 \\
\hline \multicolumn{7}{|l|}{ Postoperative fluid balance, $\mathrm{ml}$} \\
\hline Postoperative day 1 & $660(-260,2770)$ & $507(180,1200)$ & 0.689 & $670(220,2300)$ & $490(-260,1760)$ & 0.135 \\
\hline Postoperative day 2 & $100(-440,1159)$ & $318(50,720)$ & 0.319 & $320(-350,1310)$ & $100(-340,720)$ & 0.193 \\
\hline
\end{tabular}


Table 2 (continued)

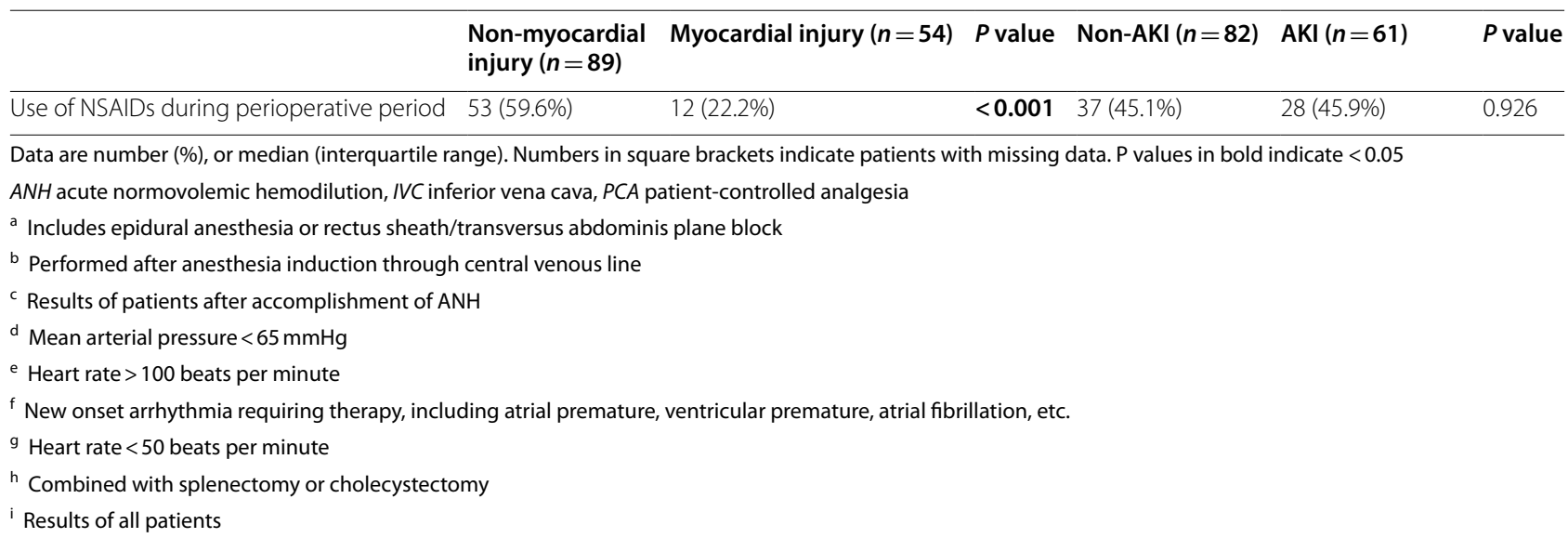

arterial pressure $<65 \mathrm{mmHg}$ ) with a median duration of 17 min (IQR 4-48) and 62.2\% developed tachycardia during surgery. All these might had led to significant myocardial injury [34-36].

In our patients, the majority of myocardial injury was silent (without ischemic symptoms, ECG changes or imaging findings) and occurred within the first day after surgery. These were in line with previous studies $[17,18$, 33]. We found that combined Mayo level III + IV was an independent risk factor of postoperative myocardial injury when compared with combined level I+ II, possibly due to more severe intraoperative hemodynamic fluctuation. In line with this, previous studies also reported that patients with higher level tumor thrombus
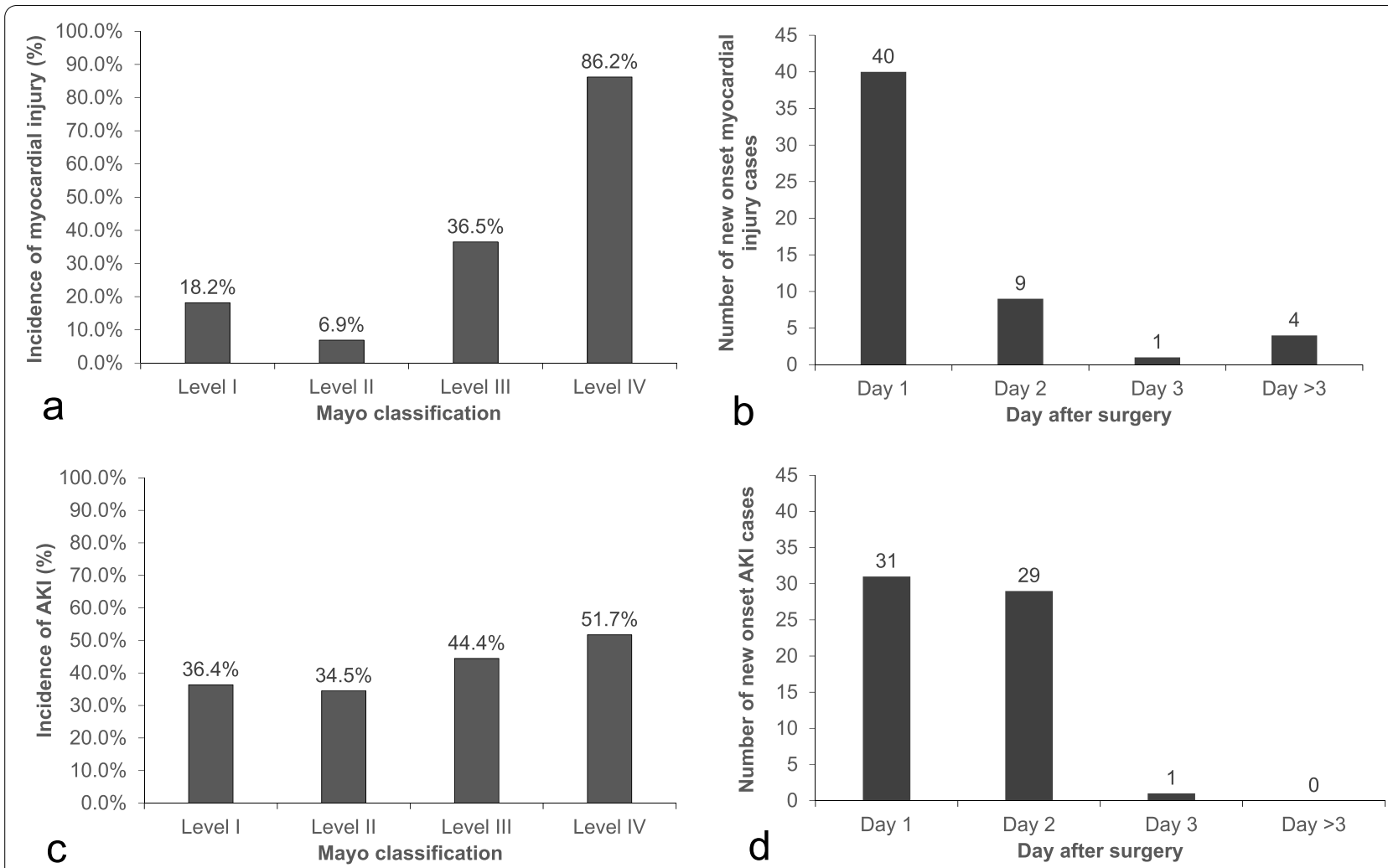

Fig. 2 Occurrence of myocardial injury according to Mayo classification level (a) and days after surgery (b). The majority of myocardial injury occurred in patients with Mayo levels III and IV and during the first day after surgery. Occurrence of acute kidney injury according to Mayo classification level (c) and days after surgery (d). The development of AKI was not related with Mayo level, but the majority occurred within the first two postoperative days 
Table 3 Postoperative outcomes

\begin{tabular}{|c|c|c|c|c|c|c|}
\hline & $\begin{array}{l}\text { Non-myocardial } \\
\text { injury }(n=89)\end{array}$ & Myocardial injury $(n=54)$ & $P$ value & Non-AKI $(n=82)$ & AKI $(n=61)$ & $P$ value \\
\hline Highest cTnl (ng/ml), median (95\%Cl) & $0.010(0.003,0.010)$ & $0.790(0.067,2.272)$ & $<0.001$ & $0.010(0.003,0.038)$ & $0.040(0.010,1.100)$ & 0.004 \\
\hline Development of myocardial injury & $0(0 \%)$ & $54(100.0 \%)$ & - & $23(28.0 \%)$ & $31(50.8 \%)$ & 0.005 \\
\hline Acute myocardial infarction ${ }^{a}$ & $0(0 \%)$ & $11(20.4 \%)$ & $<0.001$ & $3(3.7 \%)$ & $8(13.1 \%)$ & 0.036 \\
\hline Highest creatinine $(\mu \mathrm{mol} / \mathrm{L})$ & $113.0(98.4,129.4)$ & $125.0(99.0,146.3)$ & 0.152 & $106.5(92.0,123.3)$ & $129.6(116.1,160.7)$ & $<0.001$ \\
\hline Development of AKI & $30(33.7 \%)$ & $31(57.4 \%)$ & 0.005 & $0(0.0 \%)$ & $61(100.0 \%)$ & - \\
\hline Stage of AKI & & & 0.014 & & & - \\
\hline Stage 1 & $26(29.2 \%)$ & $27(50.0 \%)$ & & $0(0.0 \%)$ & $53(86.9 \%)$ & \\
\hline Stage 2 & $2(2.2 \%)$ & $4(7.4 \%)$ & & $0(0.0 \%)$ & $6(9.8 \%)$ & \\
\hline Stage 3 & $2(2.2 \%)$ & $0(0.0 \%)$ & & $0(0.0 \%)$ & $2(3.3 \%)$ & \\
\hline Requirement of RRT & $1(1.1 \%)$ & $0(0.0 \%)$ & $>0.999$ & $0(0.0 \%)$ & $1(1.6 \%)$ & 0.427 \\
\hline ICU admission & $57(64.0 \%)$ & $51(94.4 \%)$ & $<0.001$ & $59(72.0 \%)$ & $49(80.3 \%)$ & 0.249 \\
\hline With endotracheal intubation & $44(49.4 \%)$ & $49(90.7 \%)$ & $<0.001$ & $53(64.6 \%)$ & $40(65.6 \%)$ & 0.907 \\
\hline Mechanical ventilation, $\mathrm{h}$ & $0(0,0)$ & $11(8,14)$ & $<0.001$ & $4(2,6)$ & $5(3,7)$ & 0.247 \\
\hline Length of stay in ICU, h & $14(12,16)$ & $46(29,63)$ & $<0.001$ & $20(17,23)$ & $36(19,53)$ & 0.046 \\
\hline Other complications & $1(1.1 \%)$ & $13(24.1 \%)$ & 0.001 & $2(2.4 \%)$ & $12(19.7 \%)$ & 0.005 \\
\hline New onset arrhythmia ${ }^{\text {b }}$ & $0(0.0 \%)$ & $6(11.1 \%)$ & 0.005 & $1(1.2 \%)$ & $5(8.2 \%)$ & 0.102 \\
\hline Pulmonary embolism ${ }^{c}$ & $0(0.0 \%)$ & $2(3.7 \%)$ & 0.141 & $0(0.0 \%)$ & $2(3.3 \%)$ & 0.180 \\
\hline Pulmonary infection ${ }^{d}$ & $1(1.1 \%)$ & $4(7.4 \%)$ & 0.130 & $1(1.2 \%)$ & $4(6.6 \%)$ & 0.208 \\
\hline Wound infection ${ }^{\mathrm{e}}$ & $0(0 \%)$ & $1(1.9 \%)$ & 0.378 & $0(0.0 \%)$ & $1(1.6 \%)$ & 0.427 \\
\hline Length of hospital stay, day & $7(6,7)$ & $13(12,14)$ & $<0.001$ & $8(7,9)$ & $10(7,13)$ & 0.027 \\
\hline In-hospital mortality & $0(0 \%)$ & $0(0 \%)$ & - & $0(0 \%)$ & $0(0 \%)$ & - \\
\hline
\end{tabular}

Data are median (interquartile range), number (\%), or median $(95 \% \mathrm{Cl}) . P$ values in bold indicate $<0.05$

$A K I$ acute kidney injury (diagnosed according to KDIGO criteria), RRT renal replacement therapy, ICU intension care unit

a Acute myocardial infarction refers to dynamic elevations of cardiac troponin in combination with either ischemic symptoms, electrocardiogram changes, or imaging findings

b New onset arrhythmia refers to new

-onset atrial fibrillation or paroxysmal supraventricular tachycardia necessitating medical treatment

c Pulmonary embolism confirmed by computed tomography pulmonary angiography

${ }^{d}$ New infiltrate on chest radiograph with hyperthermia $\left(>38.3^{\circ} \mathrm{C}\right)$ and leukocytosis $\left(\geq 12,000 / \mathrm{mm}^{3}\right)$ and required intravenous antibiotic therapy

e Wound infection refers to pus expressed from the incision and bacteria cultured from the pus

(usually level III or higher) had increased major complications and even mortality [15, 37, 38]. Therefore, patients with IVC thrombus of level III or higher should be managed more cautiously. In our results, long duration tachycardia was another independent risk factor of myocardial injury. This could be explained by increased oxygen demand and shortened diastolic phase of the myocardium; the underlying hemodynamic changes might also contribute. The association between tachycardia and myocardial injury had been reported in other surgical patients $[35,36]$. As a potentially modifiable factor, intraoperative tachycardia should be prevented and carefully managed. We found that acute normovolemic hemodilution was an independent risk factor of myocardial injury. This may be explained by the decrease of oxygen content and delivery after hemodilution [39, 40]. Whereas $62.2 \%$ of our patients developed tachyarrhythmia which increased oxygen consumption and, thus, myocardial oxygen delivery-consumption mismatch. In line with others [41], long duration of surgery was also identified as a risk factor of myocardial injury in this cohort. On the other hand, we identified male sex as a protective factor. One possible reason was that large vessel size in male patients (compared with females) made the procedures less difficult. In accord with ours, Hassan and colleges [42] reported that female gender was an independent risk of major adverse cardiovascular events after peripheral artery disease intervention.

The incidence of AKI in our patients was much higher than in many previous studies [24, 25, 27, 28]. But our results were close to those of Shin et al. [30], the only study performed in a similar patient population, i.e., following nephrectomy with IVC thrombectomy. The high AKI incidence can be attributed to the type of surgery (radical nephrectomy) [26] and the concomitant 
Table 4 Predictors of myocardial injury

\begin{tabular}{|c|c|c|c|c|}
\hline & \multicolumn{2}{|c|}{ Univariable analysis $^{a}$} & \multicolumn{2}{|c|}{ Multivariable analysis $^{b}$} \\
\hline & OR $(95 \% \mathrm{Cl})$ & $P$ value & OR $(95 \% \mathrm{Cl})$ & $P$ value \\
\hline Age, year & $0.97(0.95,1.00)$ & 0.069 & - & - \\
\hline Male sex & $0.43(0.20,0.92)$ & 0.029 & $0.27(0.10,0.71)$ & 0.008 \\
\hline ASA class (III + IV vs. I+ II) & & & - & - \\
\hline $1+\|$ & Ref & & - & - \\
\hline$I I I+I V$ & $1.94(0.94,4.02)$ & 0.075 & - & - \\
\hline \multicolumn{5}{|l|}{ Mayo classification } \\
\hline $1+11$ & Ref & & Ref & - \\
\hline$I I I+I V$ & $8.18(3.18,21.1)$ & $<0.001$ & $4.21(1.42,12.4)$ & 0.009 \\
\hline Use of nitrous oxide during anesthesia & $0.46(0.22,0.95)$ & 0.037 & - & - \\
\hline Acute normovolemic hemodilution before surgery & $2.21(1.09,4.48)$ & 0.028 & $2.66(1.10,6.41)$ & 0.029 \\
\hline Duration of intraoperative hypotension, 20 min & $1.37(1.11,1.69)$ & 0.004 & - & - \\
\hline Duration of intraoperative tachycardia, 20 min & $1.73(1.18,2.53)$ & $<0.001$ & $1.49(1.03,2.16)$ & 0.036 \\
\hline Intraoperative bradycardia & $2.99(1.26,7.06)$ & 0.013 & - & - \\
\hline Intraoperative allogeneic blood transfusion & $5.25(2.40,11.49)$ & $<0.001$ & - & - \\
\hline Complete IVC clamping during surgery & $3.62(1.17,11.26)$ & 0.026 & - & - \\
\hline Duration of surgery, $\mathrm{h}$ & $1.71(1.37,2.12)$ & $<0.001$ & $1.48(1.03,2.16)$ & 0.009 \\
\hline Use of NSAIDs during perioperative period & $0.19(0.09,0.42)$ & $<0.001$ & - & - \\
\hline
\end{tabular}

Preoperative abnormal kidney function and Charlson Comorbidity Index were excluded because of correlation with ASA class

Intraoperative lowest hemoglobin was excluded because of correlation with intraoperative allogeneic blood transfusion

Intraoperative hypotension and intraoperative highest lactic acid were excluded because of correlation with duration of intraoperative hypotension

Intraoperative tachycardia was excluded because of correlation with duration of intraoperative tachycardia

Duration of anesthesia, volume of fluid infusion, intraoperative infusion balance and urine output were excluded because of correlation with duration of surgery

Colloid fluid, fresh frozen plasma and platelet concentrate were excluded because of correlation with the allogeneic blood transfusion

Hepatic hilum clamping and use of cardiopulmonary bypass during surgery and duration of cardiopulmonary bypass were excluded because of correlation with the Mayo classification of tumor thrombus

${ }^{\text {a }}$ Myocardial injury was modeled as a function of a single factor in the univariable logistic regression analysis

b Myocardial injury was modeled as a function of all factors with a $P$ value $<0.2$ in the univariate analysis or those that were considered clinically important. Multivariable analysis was performed using the backward method. Hosmer-Lemeshow test of goodness of fit of the model: $x^{2}=3.135, \mathrm{df}=8, P=0.926$

hemodynamic fluctuation during surgery [34, 43]. Furthermore, clamping of IVC and/or hepatic hilum during surgery might directly impair renal function [44]. In our patients, high BMI was independently associated with an increased risk of AKI. Similar association was reported in critically ill patients [45-47]. The potential mechanisms remain unclear and may include chronic inflammation associated with obesity [48]; but it is also possibly due to the method defining AKI, i.e., patients with high body mass produce more creatinine. We found that long duration of intraoperative hypotension was an independent risk factor of AKI; this was in line with previous findings $[34,49,50]$. Optimizing blood pressure management may improve outcome but requires further confirmation [51]. On the contrary, we found that preoperative renal arterial embolism was a protective factor of AKI. The potential advantages of renal artery embolism in patients with IVC tumor thrombus had been reported by others [52]. Our results provide further evidence although more studies are required.

In addition to the retrospective nature, our study had some other limitations. First, because of the rarity of the surgery, the sample size included in the study was relatively small. We might have missed some risk factors. Second, we only collected in-hospital data. Long-term outcomes of our patients remained unclear. Third, as a single-center study, the generalizability of our results may be limited. Fourth, there might be interaction between myocardial injury and AKI in our patients. However, because of limited sample size and follow-up duration, we did not evaluate heart-renal cross-talk in this study [53].

In summary, our results showed that myocardial injury and AKI were common in patients recovering from radical nephrectomy with IVC thrombectomy. Patients who developed these complications had worse perioperative outcomes. Among the potentially 
Table 5 Predictors of AKI

\begin{tabular}{|c|c|c|c|c|}
\hline & \multicolumn{2}{|c|}{ Univariable analysis $^{a}$} & \multicolumn{2}{|c|}{ Multivariable analysis ${ }^{b}$} \\
\hline & OR $(95 \% \mathrm{Cl})$ & $P$ value & OR $(95 \% \mathrm{Cl})$ & $P$ value \\
\hline Age, year ${ }^{c}$ & $1.01(0.98,1.04)$ & 0.431 & - & - \\
\hline Body mass index, $\mathrm{kg} / \mathrm{m}^{2}$ & $1.12(1.01,1.23)$ & 0.029 & $1.18(1.06,1.33)$ & 0.004 \\
\hline Previous stroke & $3.57(0.67,19.07)$ & 0.136 & - & - \\
\hline Previous surgery & $0.46(0.18,1.19)$ & 0.108 & - & - \\
\hline \multicolumn{5}{|l|}{ Mayo classification ${ }^{c}$} \\
\hline $1+\|$ & Ref & - & - & - \\
\hline$I I I+I V$ & $1.61(0.80,3.26)$ & 0.186 & - & - \\
\hline Interval from contrast-enhanced examination, day & $1.02(0.99,1.05)$ & 0.144 & - & - \\
\hline Selective renal arterial embolization & $0.40(0.16,1.02)$ & 0.056 & $0.20(0.07,0.59)$ & 0.004 \\
\hline Use of sevoflurane during anesthesia & $1.65(0.84,3.25)$ & 0.145 & - & - \\
\hline Use of dexmedetomidine during anesthesia & $0.63(0.32,1.23)$ & 0.176 & - & - \\
\hline Acute normovolemic hemodilution before surgery & $1.58(0.79,3.17)$ & 0.194 & - & - \\
\hline Duration of intraoperative hypotension, $20 \mathrm{~min}$ & $1.27(1.04,1.54)$ & 0.021 & $1.30(1.04,1.64)$ & 0.024 \\
\hline Occurrence of intraoperative tachycardia & $1.87(0.93,3.77)$ & 0.081 & - & - \\
\hline Transfusion of platelet concentrate & $2.42(0.97,6.03)$ & 0.059 & - & - \\
\hline Use of PCA after surgery & $5.60(0.67,46.78)$ & 0.112 & - & - \\
\hline
\end{tabular}

Highest lactic acid during surgery was excluded because of correlation with duration of hypotension

a Acute kidney injury was modeled as a function of a single factor in the univariable logistic regression analysis

${ }^{b}$ Acute kidney injury was modeled as a function of all factors with a $P$ value $<0.2$ in the univariate analyses or those that were considered clinically important. Multivariable analysis was performed using the backward method. Hosmer-Lemeshow test of goodness of fit of the model: $x^{2}=4.612, \mathrm{df}=8, P=0.798$

c Included because of clinical importance

modifiable factors, long-duration intraoperative tachyarrhythmia was associated with increased risk of myocardial injury; preoperative renal artery embolism was associated with a decreased risk, whereas long-duration intraoperative hypotension was associated with an increased risk of AKI. Further studies are required to investigate whether intervention targeting these factors can improve patients' outcomes.

\section{Abbreviations}

AKI: Acute kidney injury; KDIGO: Kidney Disease: Improving Global Outcomes; OR: Odd ratio; Cl: Confidence interval; RCC: Renal cell carcinoma; IVC: Inferior vena cava; ECG: Electrocardiogram; ANH: Acute normovolemic hemodilution; CPB: Cardiopulmonary bypass; ICU: Intensive care unit; CTnl: Cardiac troponin I; PCA: Patient-controlled analgesia; AMI: Acute myocardial infarction; IQR: Interquartile range; BMI: Body mass index; HR: Hazard ratio.

\section{Supplementary Information}

The online version contains supplementary material available at https://doi. org/10.1186/s12871-021-01462-y.

Additional file 1. STROBE checklist.

Additional file 2. Supplement Table. Univariable analyses of perioperative factors.

\section{Acknowledgements}

The authors gratefully acknowledge Prof. Zhi-Song He (Department of Urology, Peking University First Hospital, Beijing, China) for his help in collecting data and Ms. Xue-Ying Li (Department of Biostatistics, Peking University First Hospital, Beijing, China) for her help in statistical analysis.

\section{Authors' contributions}

Y-BH designed the study, collected, analyzed and interpreted data, and drafted the manuscript. XL designed the study, collected, analyzed and interrupted data, and drafted the manuscript. D-XW designed the study, reviewed the original data and the results of analyses, and critically revised the manuscript. All authors have read and approved the manuscript.

\section{Funding}

This work was funded by the National Key R\&D Program of China (2018YFC2001800). The sponsor has no role in the study design and conduct; the collection, management, analysis, and interpretation of the data; or the preparation and approval of the manuscript.

\section{Availability of data and materials}

The datasets used and analyzed in the current study are available from the corresponding author upon reasonable request.

\section{Declarations}

\section{Ethics approval and consent to participate}

This study was approved by the Biomedical Research Ethics Committee of Peking University First Hospital (2019-205). The ethics committee agreed to waive written informed consent.

\section{Consent for publication}

Not applicable.

Competing interests

The authors declare no conflicts of interest. 


\section{Author details}

${ }^{1}$ Department of Anesthesiology and Critical Care Medicine, Peking University First Hospital, Beijing 100034, China. ${ }^{2}$ Outcomes Research Consortium, Cleveland, OH, USA.

Received: 16 March 2021 Accepted: 30 September 2021 Published online: 12 October 2021

\section{References}

1. Ali AS, Vasdev N, Shanmuganathan S, Paez E, Dark JH, Manas D, et al. The surgical management and prognosis of renal cell cancer with IVC tumor thrombus: 15-years of experience using a multi-specialty approach at a single UK referral center. Urol Oncol. 2013;31(7):1298-304.

2. Bissada NK, Yakout HH, Babanouri A, Elsalamony T, Fahmy W, Gunham M, et al. Long-term experience with management of renal cell carcinoma involving the inferior vena cava. Urology. 2003;61(1):89-92.

3. Kaplan S, Ekici S, Dogan R, Demircin M, Ozen H, Pasaoglu I. Surgical management of renal cell carcinoma with inferior vena cava tumor thrombus. Am J Surg. 2002;183(3):292-9.

4. Martinez-Salamanca Jl, Huang WC, Millan I, Bertini R, Bianco FJ, Carballido JA, et al. Prognostic impact of the 2009 UICC/AJCC TNM staging system for renal cell carcinoma with venous extension. Eur Urol. 2011;59(1):120-7.

5. Blute ML, Leibovich BC, Lohse CM, Cheville JC, Zincke H. The Mayo Clinic experience with surgical management, complications and outcome for patients with renal cell carcinoma and venous tumour thrombus. BJU Int. 2004:94(1):33-41.

6. Ciancio G, Manoharan M, Katkoori D, De Los SR, Soloway MS. Longterm survival in patients undergoing radical nephrectomy and inferior vena cava thrombectomy: single-center experience. Eur Urol. 2010;57(4):667-72.

7. Haferkamp A, Bastian PJ, Jakobi H, Pritsch M, Pfitzenmaier J, Albers P, et al. Renal cell carcinoma with tumor thrombus extension into the vena cava: prospective long-term followup. J Urol. 2007;177(5):1703-8.

8. Tang Q, Song Y, Li XS, Zhang CJ, Cai L, Song G, et al. Zhou LQ: [surgical management and long-term outcomes of patients with renal cell carcinoma accompanied with venous tumor thrombus]. Beijing Da Xue Xue Bao. 2013;45(4):549-53.

9. Chen K, Wang J, Dai J, Luo A, Tian Y, Guan Z, et al. Anesthetic management of radical nephrectomy in patients with renal cell carcinoma involving renal vein or inferior vena cava. Tumori. 2019;105(5):411-6.

10. Novick AC, Kaye MC, Cosgrove DM, Angermeier K, Pontes JE, Montie $J E$, et al. Experience with cardiopulmonary bypass and deep hypothermic circulatory arrest in the management of retroperitoneal tumors with large vena caval thrombi. Ann Surg. 1990;212(4):472-6 discussion 476-477.

11. Stewart JR, Carey JA, McDougal WS, Merrill WH, Koch MO, Bender HJ. Cavoatrial tumor thrombectomy using cardiopulmonary bypass without circulatory arrest. Ann Thorac Surg. 1991;51(5):717-21 discussion 721-712.

12. Welz A, Schmeller N, Schmitz C, Reichart B, Hofstetter A. Resection of hypernephromas with vena caval or right atrial tumor extension using extracorporeal circulation and deep hypothermic circulatory arrest: a multidisciplinary approach. Eur J Cardiothorac Surg. 1997;12(1):127-32.

13. Lue K, Russell CM, Fisher J, Kurian T, Agarwal G, Luchey A, et al. Predictors of postoperative complications in patients who undergo radical nephrectomy and IVC Thrombectomy: a large contemporary tertiary center analysis. Clin Genitourin Cancer. 2016;14(1):89-95.

14. Vergho DC, Loeser A, Kocot A, Spahn M, Riedmiller H. Tumor thrombus of inferior vena cava in patients with renal cell carcinoma - clinical and oncological outcome of 50 patients after surgery. BMC Res Notes. 2012;5:5.

15. Abel EJ, Thompson RH, Margulis V, Heckman JE, Merril MM, Darwish $\mathrm{OM}$, et al. Perioperative outcomes following surgical resection of renal cell carcinoma with inferior vena cava thrombus extending above the hepatic veins: a contemporary multicenter experience. Eur Urol. 2014;66(3):584-92.

16. Thygesen K, Alpert JS, Jaffe AS, Chaitman BR, Bax JJ, Morrow DA, et al. Fourth universal definition of myocardial infarction (2018). J Am Coll Cardiol. 2018;72(18):2231-64.
17. Botto F, Alonso-Coello P, Chan MT, Villar JC, Xavier D, Srinathan S, et al. Myocardial injury after noncardiac surgery: a large, international, prospective cohort study establishing diagnostic criteria, characteristics, predictors, and 30-day outcomes. Anesthesiology. 2014;120(3):564-78.

18. Puelacher C, Lurati BG, Seeberger D, Sazgary L, Marbot S, Lampart A, et al. Perioperative myocardial injury after noncardiac surgery: incidence, mortality, and characterization. Circulation. 2018;137(12):1221-32.

19. Sessler DI, Khanna AK. Perioperative myocardial injury and the contribution of hypotension. Intensive Care Med. 2018;44(6):811-22.

20. Devereaux PJ, Biccard BM, Sigamani A, Xavier D, Chan M, Srinathan SK, et al. Association of Postoperative High-Sensitivity Troponin Levels with Myocardial Injury and 30-day mortality among patients undergoing noncardiac surgery. JAMA. 2017;317(16):1642-51.

21. van Waes JA, Grobben RB, Nathoe HM, Kemperman H, de Borst GJ, Peelen LM, et al. One-year mortality, causes of death, and cardiac interventions in patients with postoperative myocardial injury. Anesth Analg. 2016;123(1):29-37.

22. Thomas ME, Blaine C, Dawnay A, Devonald MA, Ftouh S, Laing C, et al. The definition of acute kidney injury and its use in practice. Kidney Int. 2015;87(1):62-73.

23. Antonelli A, Allinovi M, Cocci A, Russo Gl, Schiavina R, Rocco B, et al. The predictive role of biomarkers for the detection of acute kidney injury after partial or radical nephrectomy: a systematic review of the literature. Eur Urol Focus. 2020;6(2):344-53.

24. Long TE, Helgason D, Helgadottir S, Palsson R, Gudbjartsson T, Sigurdsson $\mathrm{GH}$, et al. Acute kidney injury after abdominal surgery: incidence, risk factors, and outcome. Anesth Analg. 2016;122(6):1912-20.

25. O'Connor ME, Kirwan CJ, Pearse RM, Prowle JR. Incidence and associations of acute kidney injury after major abdominal surgery. Intensive Care Med. 2016;42(4):521-30.

26. Kim NY, Chae D, Lee J, Kang B, Park K, Kim SY. Development of a risk scoring system for predicting acute kidney injury after minimally invasive partial and radical nephrectomy: a retrospective study. Surg Endosc. 2021;35(4):1626-35.

27. Gameiro J, Neves JB, Rodrigues N, Bekerman C, Melo MJ, Pereira M, et al. Acute kidney injury, long-term renal function and mortality in patients undergoing major abdominal surgery: a cohort analysis. Clin Kidney J. 2016:9(2):192-200.

28. Grams ME, Sang Y, Coresh J, Ballew S, Matsushita K, Molnar MZ, et al. Acute kidney injury after major surgery: a retrospective analysis of veterans health administration data. Am J Kidney Dis. 2016;67(6):872-80.

29. Kim WH, Shin KW, Ji SH, Jang YE, Lee JH, Jeong CW, et al. Robust association between acute kidney injury after radical nephrectomy and Longterm renal function. J Clin Med. 2020;9(3):619.

30. Shin S, Han Y, Park H, Chung YS, Ahn H, Kim CS, et al. Risk factors for acute kidney injury after radical nephrectomy and inferior vena cava thrombectomy for renal cell carcinoma. J Vasc Surg. 2013;58(4):1021-7.

31. Gross JB. Estimating allowable blood loss: corrected for dilution. Anesthesiology. 1983;58(3):277-80.

32. Kellum JA, Lameire N, Group KAGW. Diagnosis, evaluation, and management of acute kidney injury: a KDIGO summary (part 1). Crit Care. 2013;17(1):204.

33. Huang S, Apinyachon W, Agopian VG, Wray CL, Busuttil RW, Steadman $\mathrm{RH}$, et al. Myocardial injury in patients with hemodynamic derangements during and/or after liver transplantation. Clin Transpl. 2016;30(12):1552-7.

34. Salmasi V, Maheshwari K, Yang D, Mascha EJ, Singh A, Sessler DI, et al. Relationship between intraoperative hypotension, defined by either reduction from baseline or absolute thresholds, and acute kidney and myocardial injury after noncardiac surgery: a retrospective cohort analysis. Anesthesiology. 2017;126(1):47-65

35. Abbott T, Pearse RM, Archbold RA, Ahmad T, Niebrzegowska E, Wragg A, et al. A prospective international multicentre cohort study of intraoperative heart rate and systolic blood pressure and myocardial injury after noncardiac surgery: results of the VISION study. Anesth Analg. 2018;126(6):1936-45.

36. Abbott TE, Ackland GL, Archbold RA, Wragg A, Kam E, Ahmad T, et al. Preoperative heart rate and myocardial injury after non-cardiac surgery: results of a predefined secondary analysis of the VISION study. $\mathrm{Br} J$ Anaesth. 2016;117(2):172-81.

37. Alsina AE, Wind D, Kumar A, Rogers E, Buggs J, Bukkapatnam R, et al. Outcomes in renal cell carcinoma with IVC Thrombectomy: a multiteam 
analysis between an $\mathrm{NCl}$-designated Cancer center and a quaternary care teaching hospital. Am Surg. 2020;86(8):1005-9.

38. Ralla B, Adams L, Maxeiner A, Mang J, Krimphove M, Dushe S, et al. Perioperative and oncologic outcome in patients treated for renal cell carcinoma with an extended inferior vena cava tumour thrombus level II-IV. Aktuelle Urol. 2019. https://doi.org/10.1055/a-0919-4043.

39. Ellenberger C, Licker M. Acute normovolemic hemodilutlon: advantages and limitations. Rev Med Suisse. 2006;2(88):2670-3.

40. Ickx BE, Rigolet M, Van Der Linden PJ. Cardiovascular and metabolic response to acute normovolemic anemia. Effects of anesthesia. Anesthesiology. 2000;93(4):1011-6.

41. Duceppe E, Studzinska D, Devereaux PJ, Polok K, Gajdosz A, Lewandowski $K$, et al. Incidence and predictors of myocardial and kidney injury following endovascular aortic repair: a retrospective cohort study. Can J Anaesth. 2019;66(11):1338-46.

42. Hassan A, Abugroun A, Daoud H, Mahmoud S, Awadalla S, Volgman $A$, et al. Impact of gender differences on outcomes of peripheral artery disease intervention (from a Nationwide sample). Am J Cardiol. 2021;141:127-32.

43. Sarkar D, Agrawal A, Pal DK. Clinical assessment of stabilisation of renal function after nephrectomy. Urologia. 2021;88(3):223-6.

44. Khosravi MB, Jalaeian H, Lahsaee M, Ghaffaripour S, Salahi H, Bahador A, et al. The effect of clamping of inferior vena cava and portal vein on urine output during liver transplantation. Transplant Proc. 2007;39(4):1197-8.

45. Druml W, Metnitz B, Schaden E, Bauer P, Metnitz PG. Impact of body mass on incidence and prognosis of acute kidney injury requiring renal replacement therapy. Intensive Care Med. 2010;36(7):1221-8.

46. Shashaty MG, Meyer NJ, Localio AR, Gallop R, Bellamy SL, Holena DN, et al. African American race, obesity, and blood product transfusion are risk factors for acute kidney injury in critically ill trauma patients. J Crit Care. 2012;27(5):496-504.
47. Soto GJ, Frank AJ, Christiani DC, Gong MN. Body mass index and acute kidney injury in the acute respiratory distress syndrome. Crit Care Med. 2012;40(9):2601-8

48. Honiden S, McArdle JR. Obesity in the intensive care unit. Clin Chest Med. 2009:30(3):581-99x.

49. Sun LY, Wijeysundera DN, Tait GA, Beattie WS. Association of intraoperative hypotension with acute kidney injury after elective noncardiac surgery. Anesthesiology. 2015;123(3):515-23.

50. Walsh M, Devereaux PJ, Garg AX, Kurz A, Turan A, Rodseth RN, et al. Relationship between intraoperative mean arterial pressure and clinical outcomes after noncardiac surgery: toward an empirical definition of hypotension. Anesthesiology. 2013;119(3):507-15.

51. Futier E, Lefrant JY, Guinot PG, Godet T, Lorne E, Cuvillon P, et al. Effect of individualized vs standard blood pressure management strategies on postoperative organ dysfunction among high-risk patients undergoing major surgery: a randomized clinical trial. JAMA. 2017;318(14):1346-57.

52. Schwartz MJ, Smith EB, Trost DW, Vaughan EJ. Renal artery embolization: clinical indications and experience from over 100 cases. BJU Int. 2007;99(4):881-6.

53. Rangaswami J, Bhalla V, Blair JEA, Chang TI, Costa S, Lentine KL, et al. Cardiorenal syndrome: classification, pathophysiology, diagnosis, and treatment strategies: a scientific statement from the American Heart Association. Circulation. 2019;139(16):e840-78.

\section{Publisher's Note}

Springer Nature remains neutral with regard to jurisdictional claims in published maps and institutional affiliations.
Ready to submit your research? Choose BMC and benefit from:

- fast, convenient online submission

- thorough peer review by experienced researchers in your field

- rapid publication on acceptance

- support for research data, including large and complex data types

- gold Open Access which fosters wider collaboration and increased citations

- maximum visibility for your research: over $100 \mathrm{M}$ website views per year

At BMC, research is always in progress.

Learn more biomedcentral.com/submissions 